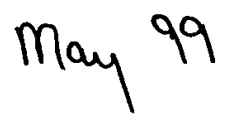

\title{
Automatic Detection of Radar Signature Defects
}

\author{
Nancy Cheadle, Naval Air Warfare Center, \\ Dennis Tackett, Modern Technology Solutions, Inc., \\ Robert Pierce, Naval Surface Warfare Center and \\ Raymond de Lacaze, Knowledge Technologies International
}

\begin{abstract}
Field-level maintenance of radar signature treatment requires that non-specialist military personnel properly identify needed repairs. To simplify this task, an automated method is required that can compare radar signature data to baseline data, measure the differences, and identify the source of serious defects. Significant work has been done using artificial intelligence (AI) techniques to simplify this diagnostic task. A portable measurement radar was used to gather signature data on a small MQM-107D target drone. One set of data was collected of a baseline vehicle. Then data was collected after several anomalies were introduced, such as an uncovered pitot tube, wing joint untaped, or fastener screw not tightened. The data was processed as global downrange plots, and then baseline data was subtracted from anomaly data and the difference was compared to signature specifications as a function of angle. AI was used to identify signature defects that require repair. The results showed that an AI-aided diagnostic tool could help identify places where signature treatment repair was needed. This tool can be adapted to a variety of user and target needs.
\end{abstract}

\section{Introduction}

Low Observable (LO) systems require specialized measurement equipment to ensure the LO characteristics are sustained. A portable diagnostic measurement system to assist maintainers of LO aircraft is required to provide measurement capability to meet the In-Service radar cross section (RCS) measurement framework, assess mission readiness and capability, and provide near real-time results. Defect isolation, diagnosis and RCS assessment are important attributes of the signature maintenance function. The purpose of this effort, managed by the Advanced Technology Test Team (ATTT), was to develop a system, which could measure an item and determine the signature health of that item with minimal human interaction. The desired features include a single system with organic measurement and analysis capability, a system that is adaptive to various users and $\mathrm{LO}$ assessment needs, and the ability to use a variety of methods to determine corrective actions. This paper provides the details of the development of automatic anomaly detection software.

\section{ICON Downrange Profile System}

The Icon Downrange Profile system is an anomaly detection and registration program based on the analysis of downrange profiles. The software provides graphical facilities for loading reference and target data, finding differences between the latter, building data abstractions of these differences and automatically matching the differences with known anomalies. Users can also register newly encountered differences. The system allows graphical retrieval and comparison of registered anomalies. The differencing and matching phases of the analysis are complex processes that use clustering algorithms to create abstract representations of the differences (known as clusters) which can be registered into an anomaly database. Menus are provided to allow users to finetune the various processing parameters in each of the phases of the analysis. The 
clustering techniques were very successful in recognizing differences and providing the necessary data abstraction paradigms for correctly retrieving known anomalies from the database and distinguishing between them. The Icon system exploits Knowledge Technologies' $G B B^{\mathrm{TM}}$ and ChalkBox ${ }^{\mathrm{TM}}$ products.

Figure 1 outlines the phases involved in data analysis. The end result is either a match with a known anomaly(s) or the option to register a newly detected type of anomaly. There are three major phases: the image analysis phase, the cluster analysis phase and the anomaly detection/registration phase. Each of these phases takes place at a different level of abstraction.

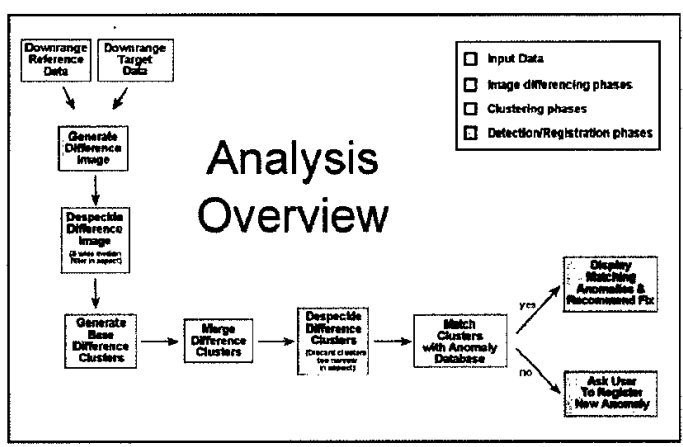

Figure 1. Analysis Overview.

Each of these steps has proven crucial to the overall process. The image analysis phases provide data alignment and noise elimination. The clustering phases provide not only data abstraction, but also a significant amount of data compression. The anomaly detection phases rely on advanced matching facilities.

The Icon analysis phases comprise three levels of data abstraction. These are the data-point level, the cluster level and the anomaly (composite cluster) level. Figure 2 outlines the three semantic levels and indicates which analysis activities are performed at each. Notice for example that there is a despeckling phase at both the datapoint level and the cluster level of the overall analysis.
At the data-point level, operations are performed on individual points in the downrange profile. At the cluster level, groups of data points (clusters) are created and operations are performed on individual clusters. Finally at the anomaly level, groups of clusters (set-composite clusters) are created as composite objects. At this level, operations are performed on groups of clusters.

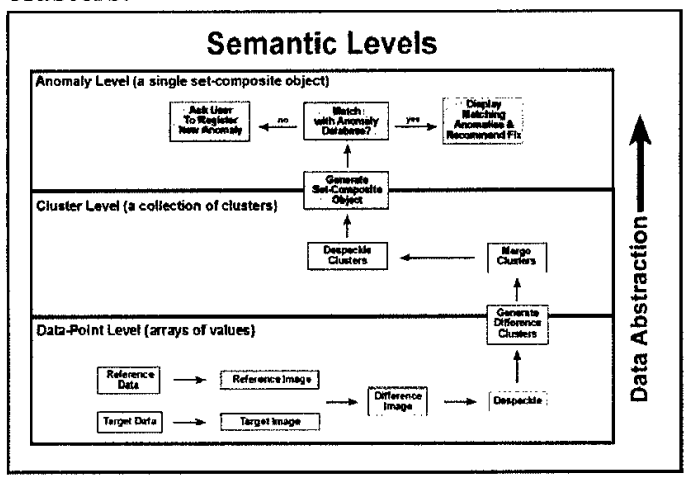

Figure 2. Data Abstraction Levels.

\section{Example/Software Validation}

To validate the usefulness of the software, measurements were taken on the MQM-107 target drone. Measurements were taken in a "pristine" condition and then realistic defects were implanted on the vehicle. The MQM-107 is shown in Figure 3. As an example of the detection process the port access screws were not secured as shown in Figure 4.

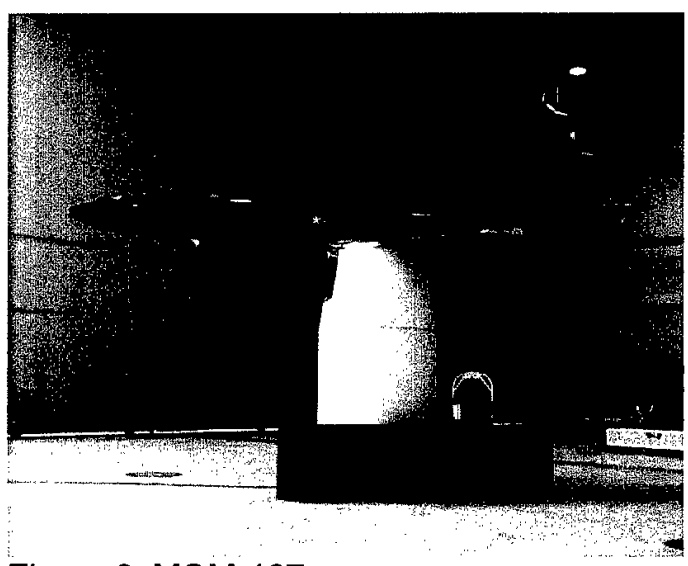

Figure 3. MQM-107 


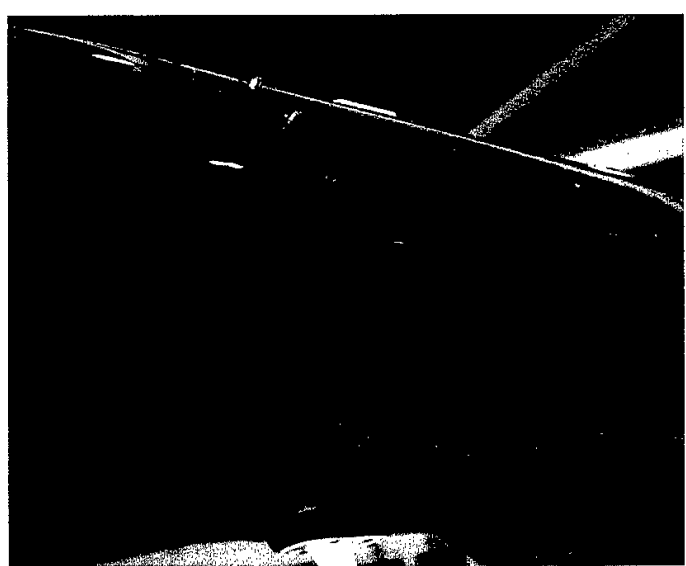

Figure 4. Access Screw Anomaly

Figure 5 presents the output of the anomaly detection process. As can been seen, in the difference data frame, the software successfully found the anomaly.

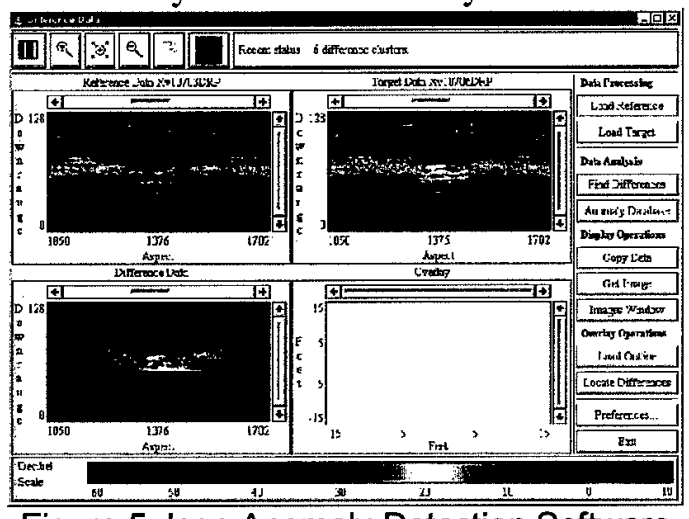

Figure 5. Icon Anomaly Detection Software Output.

\section{Summary}

This initial effort of developing automatic detection of radar signature defects was very successful. Distinguishable differences have been detected and clusters generated coincided with perceived regions of differences in the target data. Future efforts will include development of a more extensive anomaly database, extending and optimizing the clustering techniques, adapting the Icon software to ISAR data, and automating the parameter selection process.

\section{Sponsoring Agency Acknowledgment}

This effort was sponsored by the Office of Naval Research and managed by Advanced Technology Test Team (ATTT). 

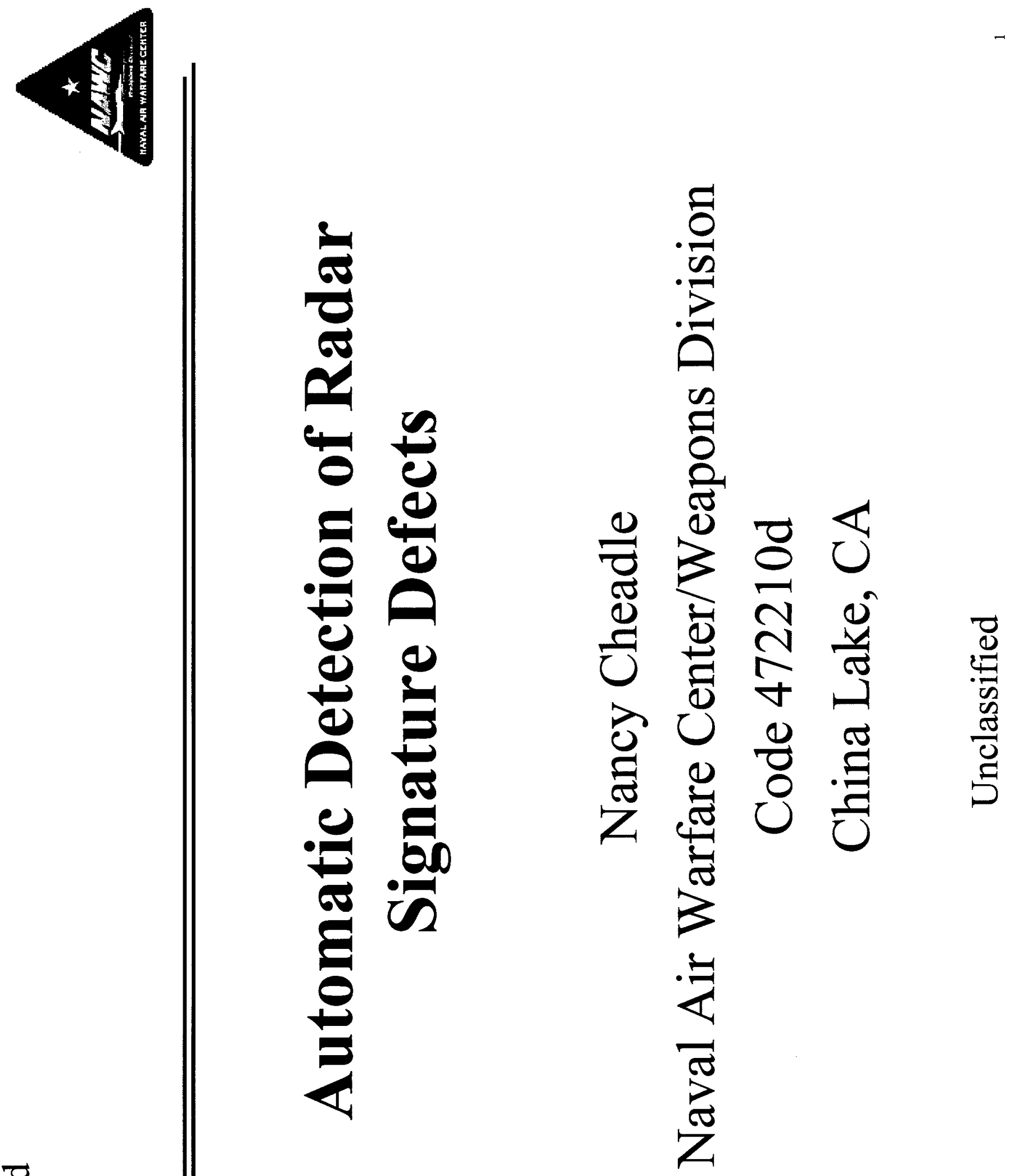

攵

$-\frac{\pi}{0}$

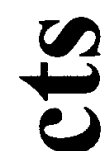

$\infty$

$\infty$

(4)

एक

60

$B$

年

0

4

$\overrightarrow{0}$

6

5 


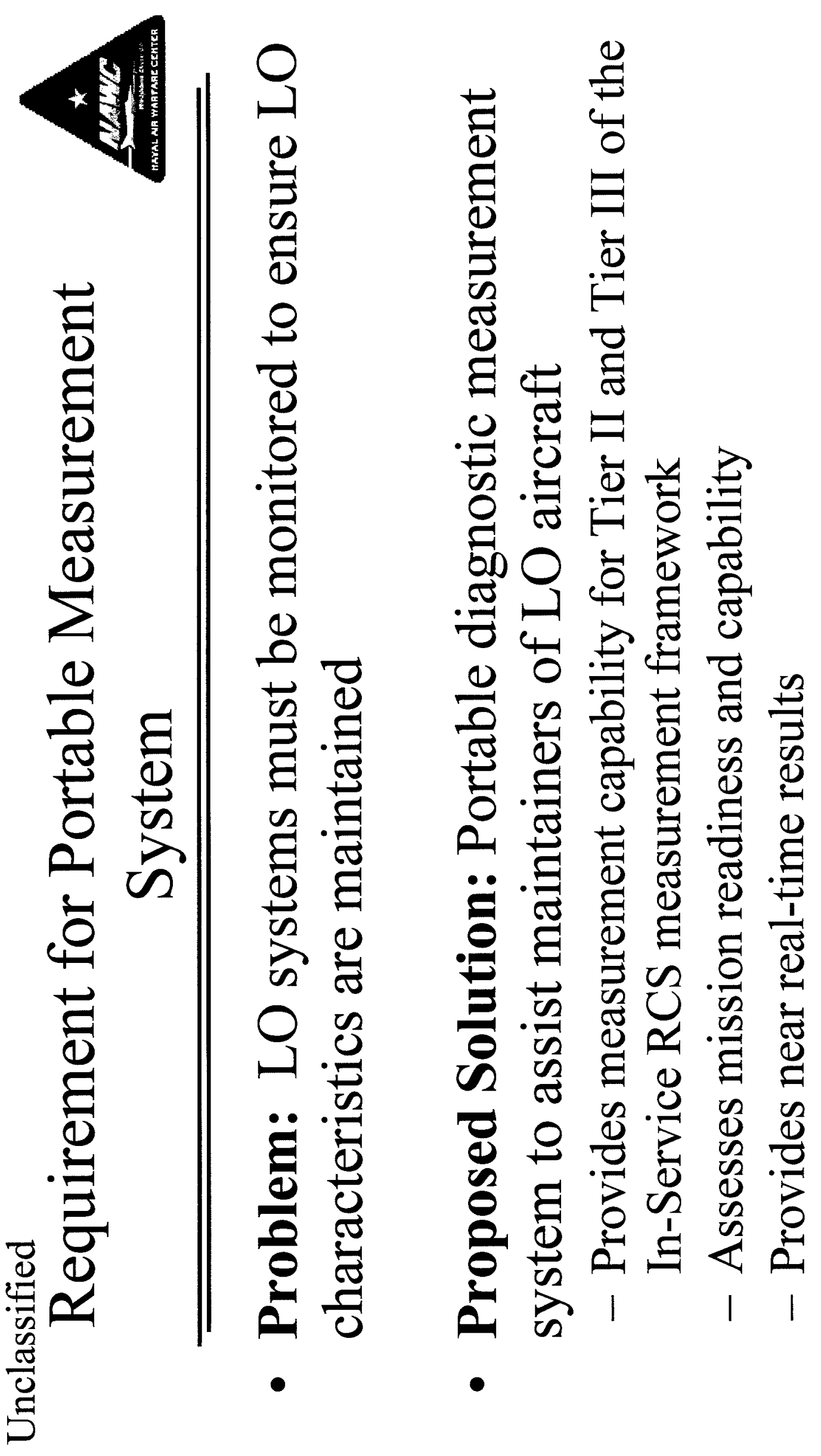

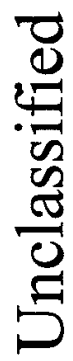




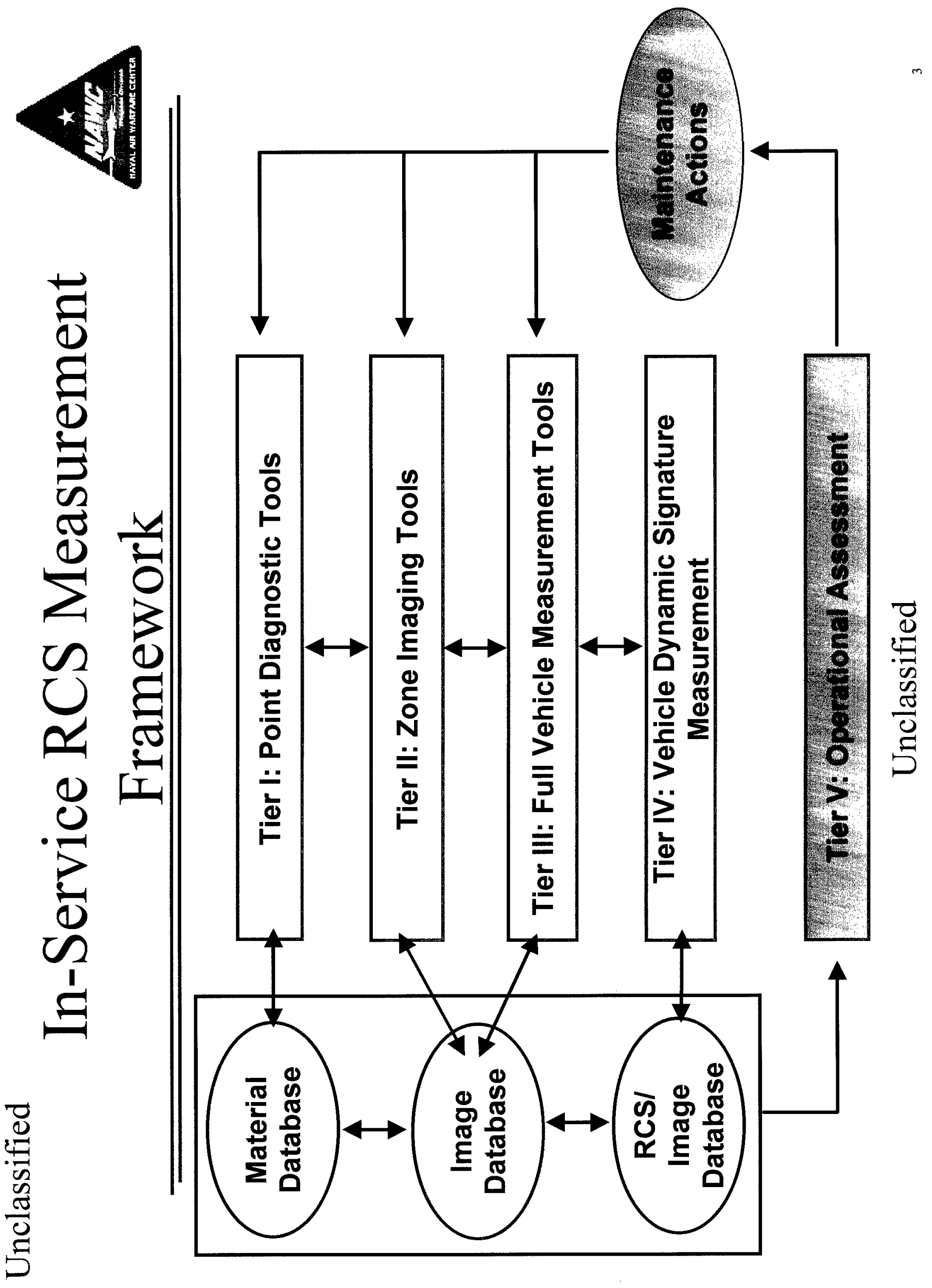




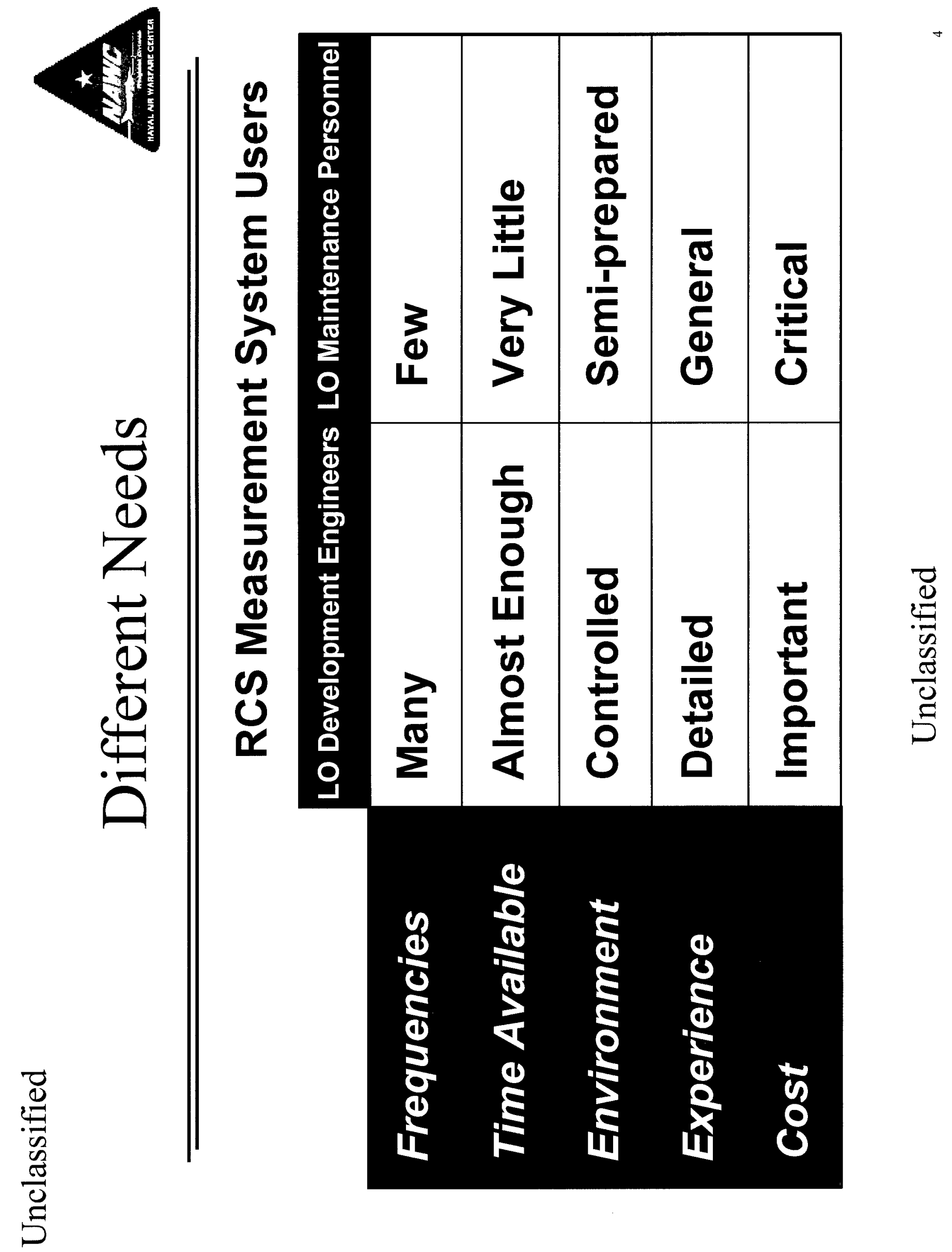




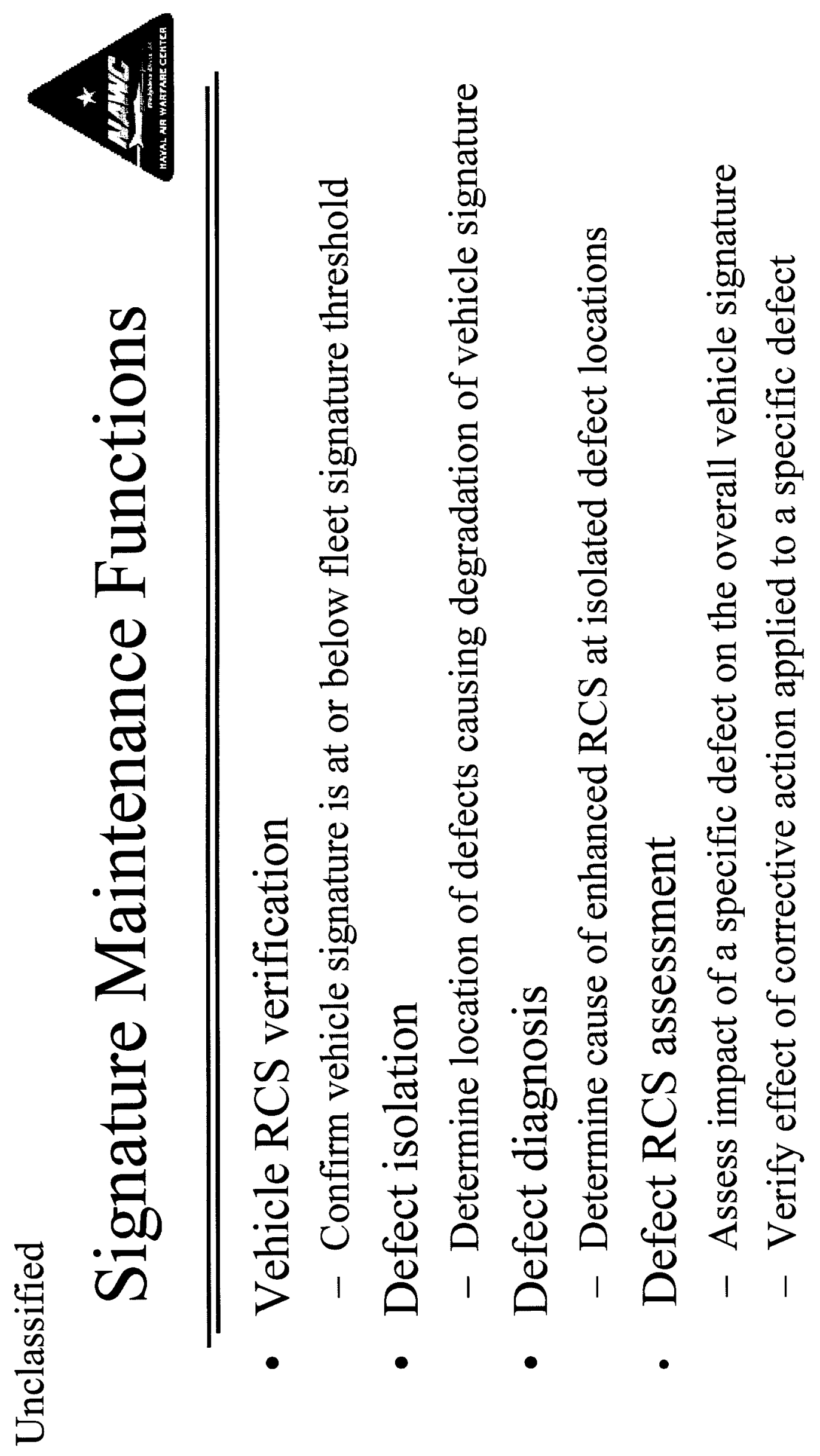

0
0
0
0
0
0
0
0
0 


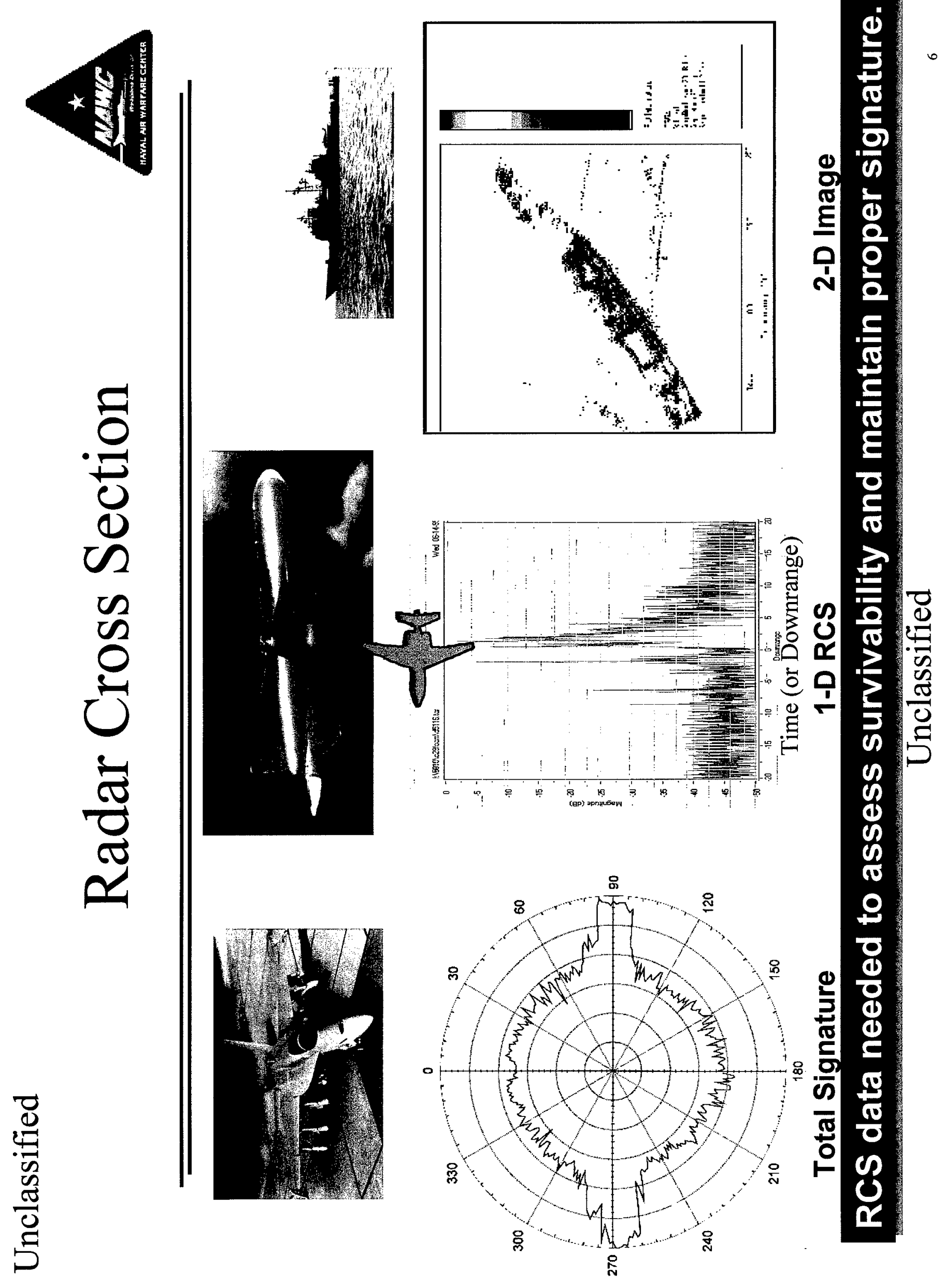




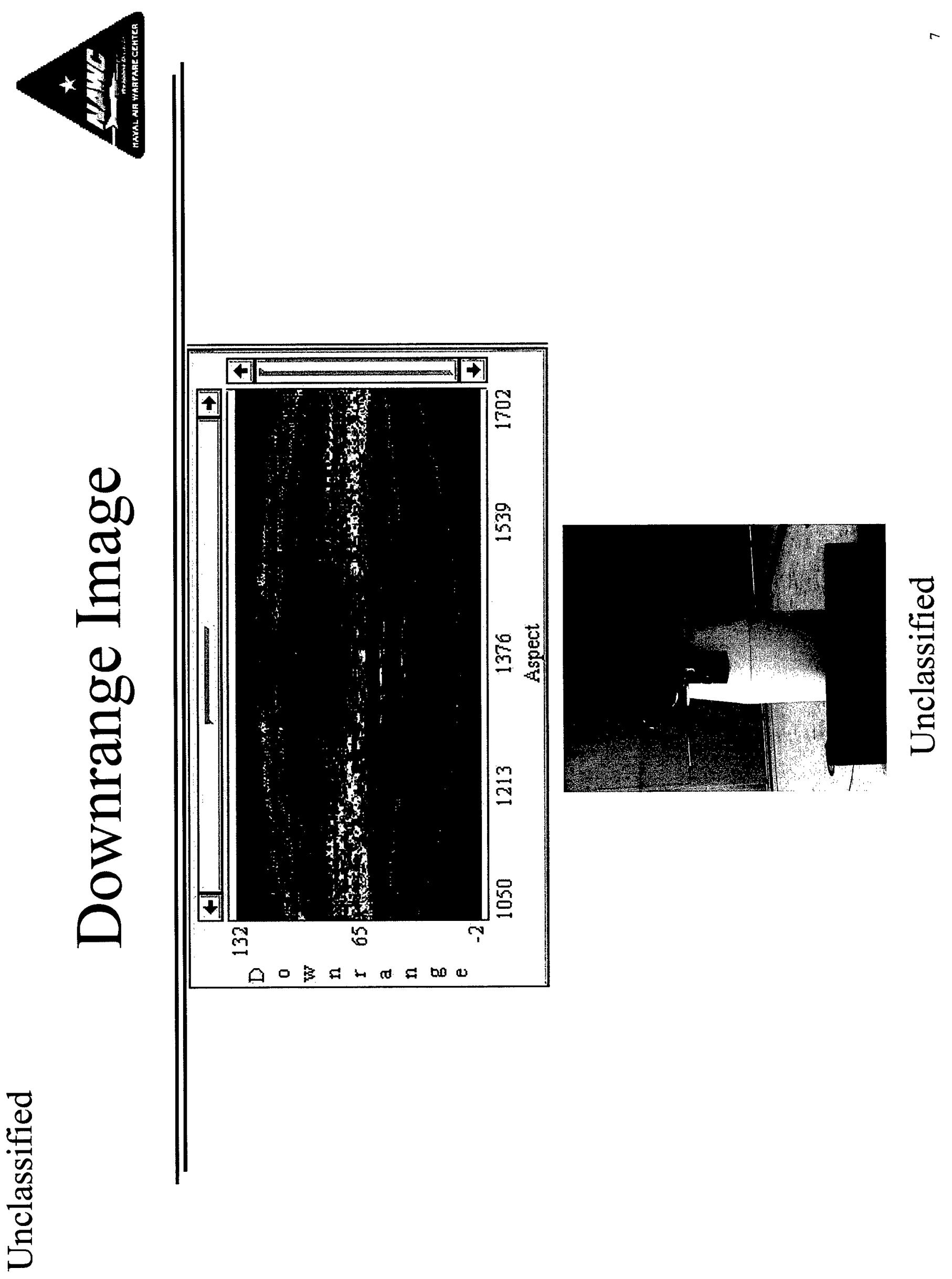




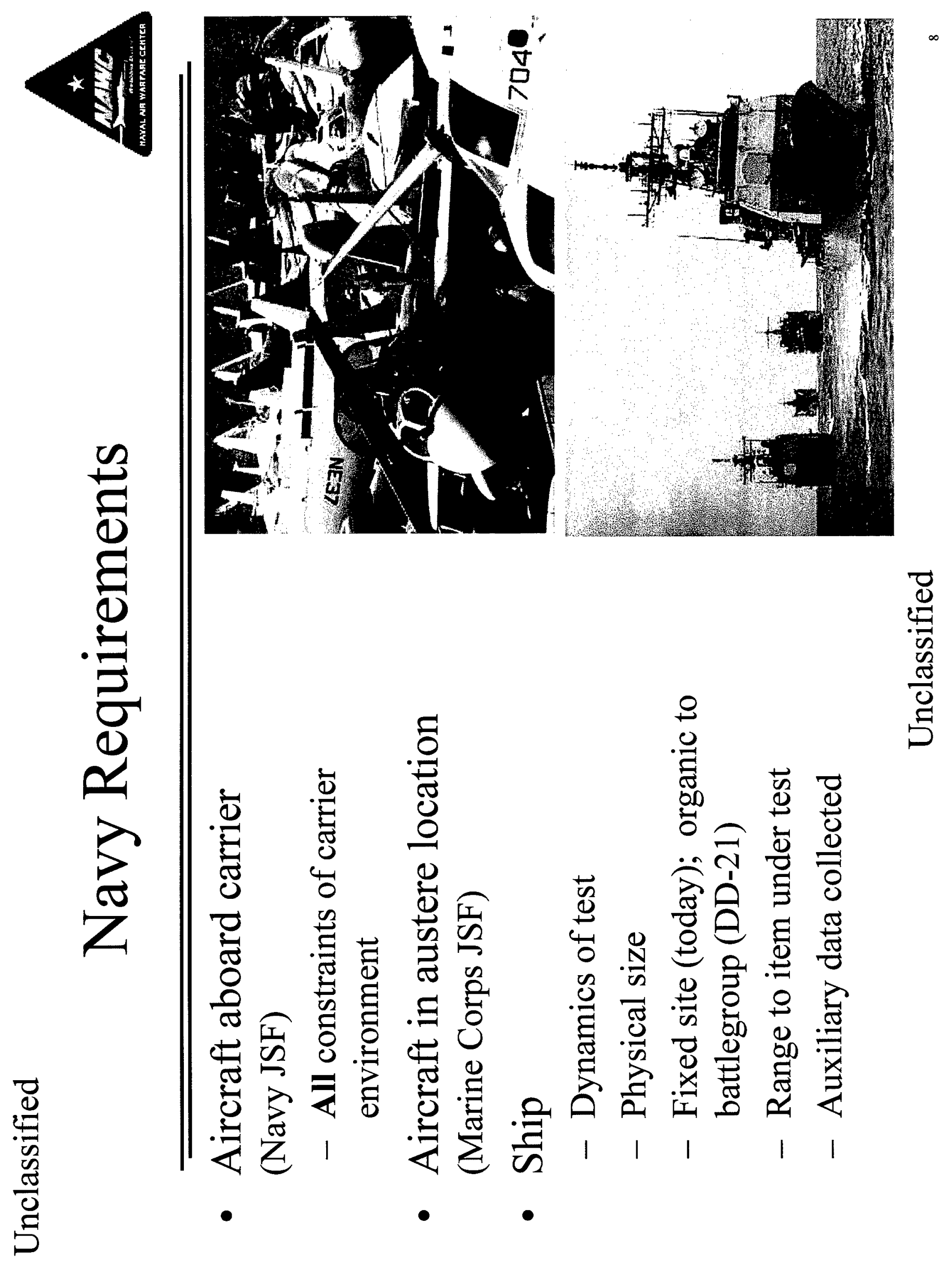



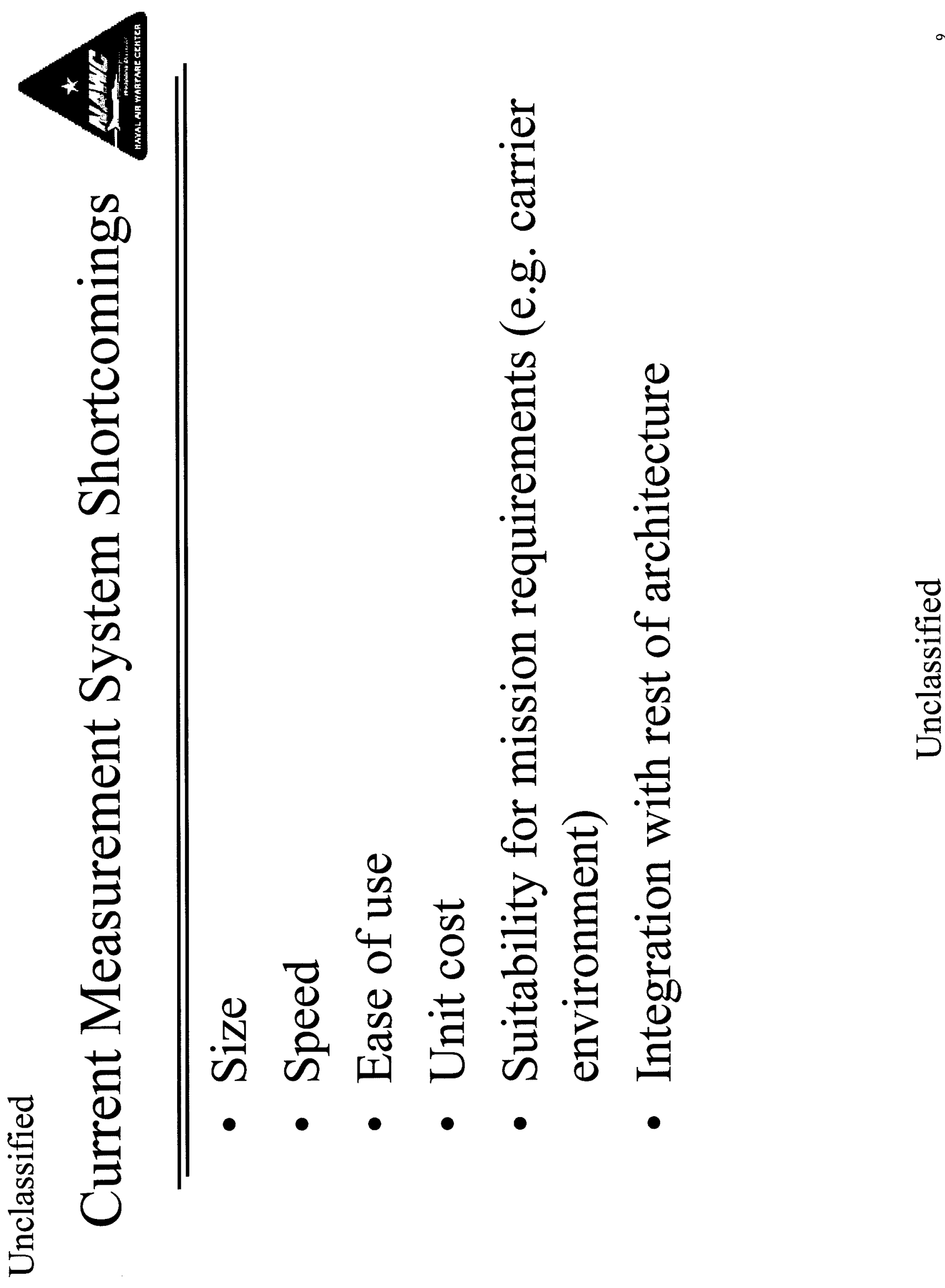


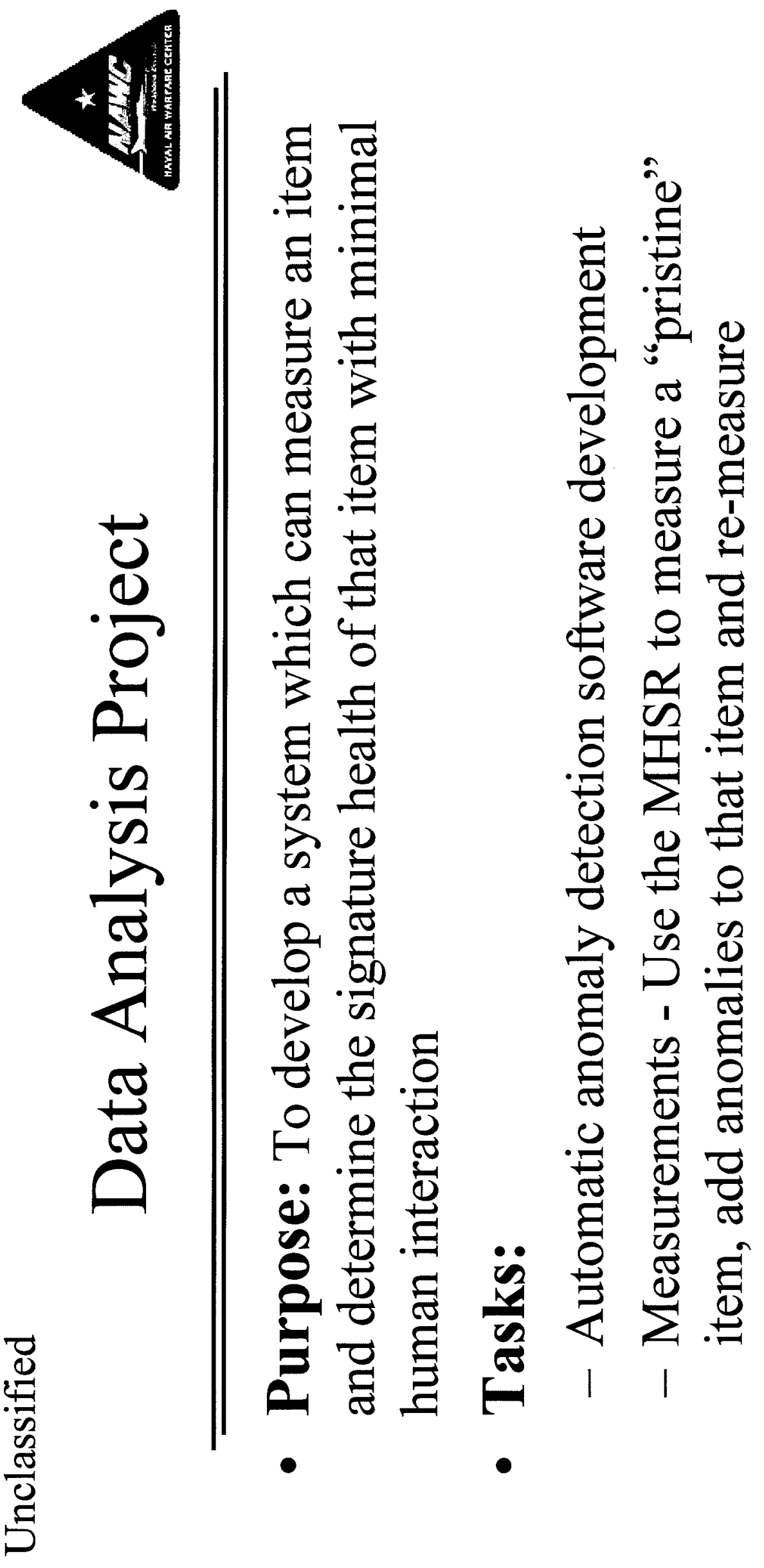




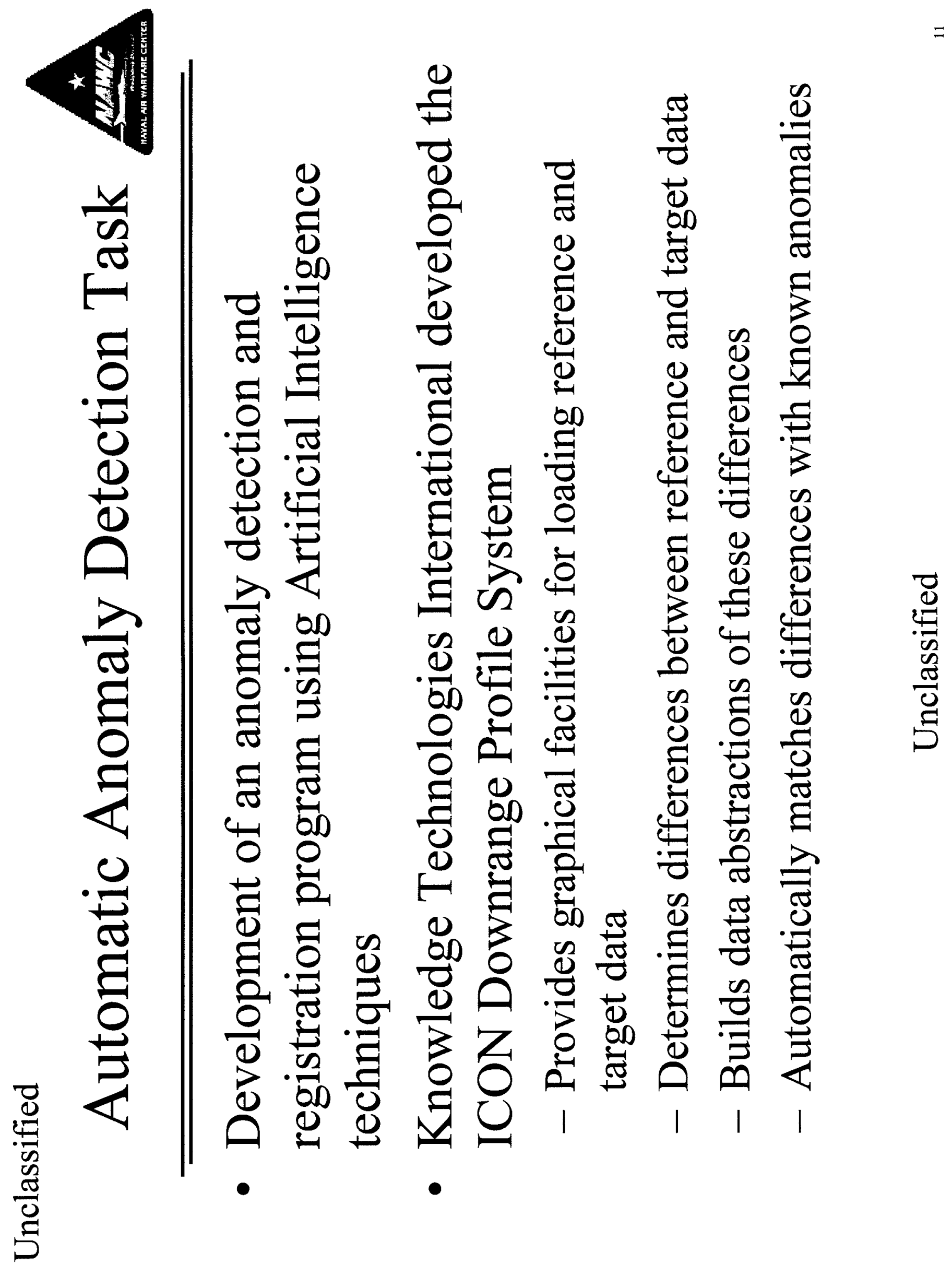




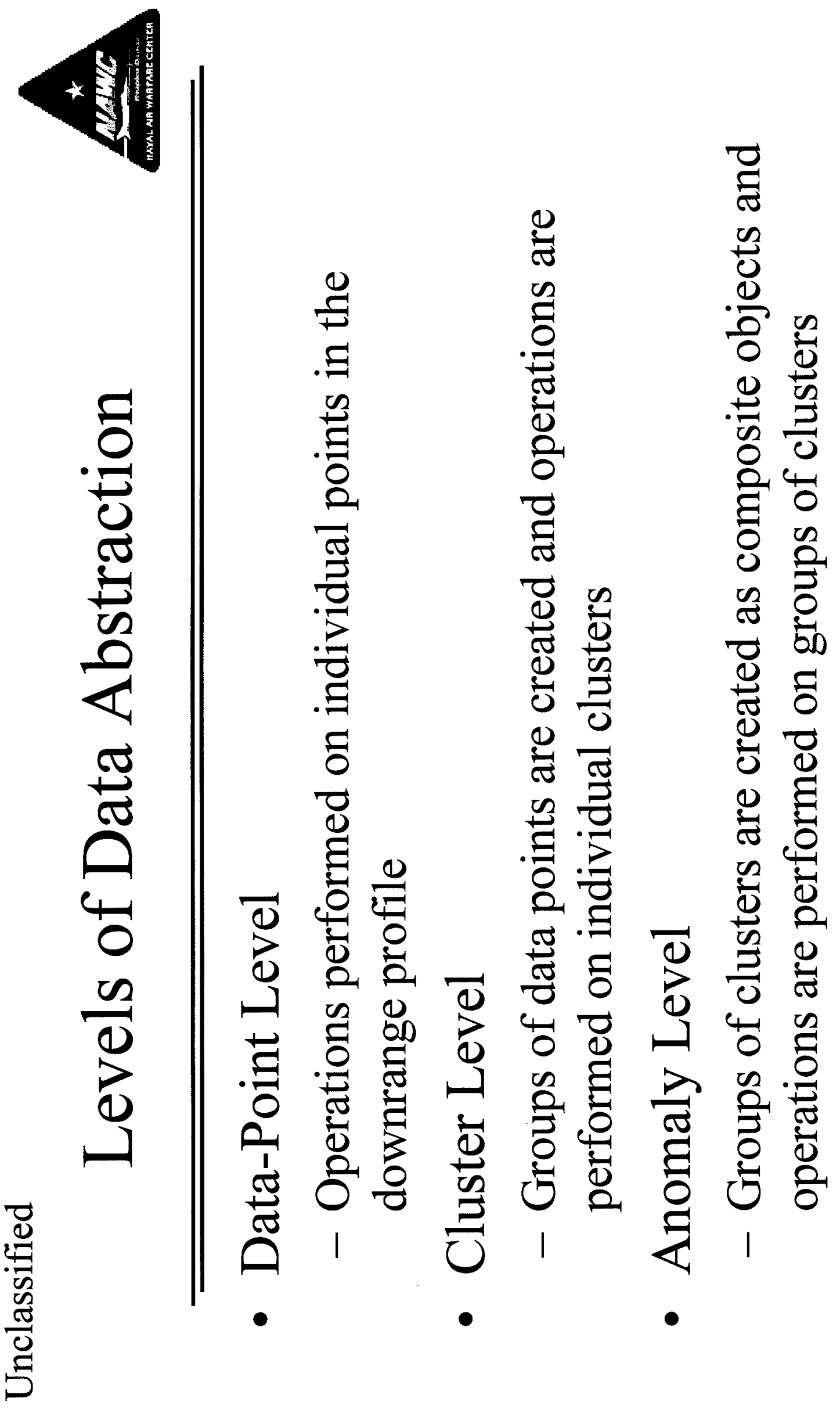

足 


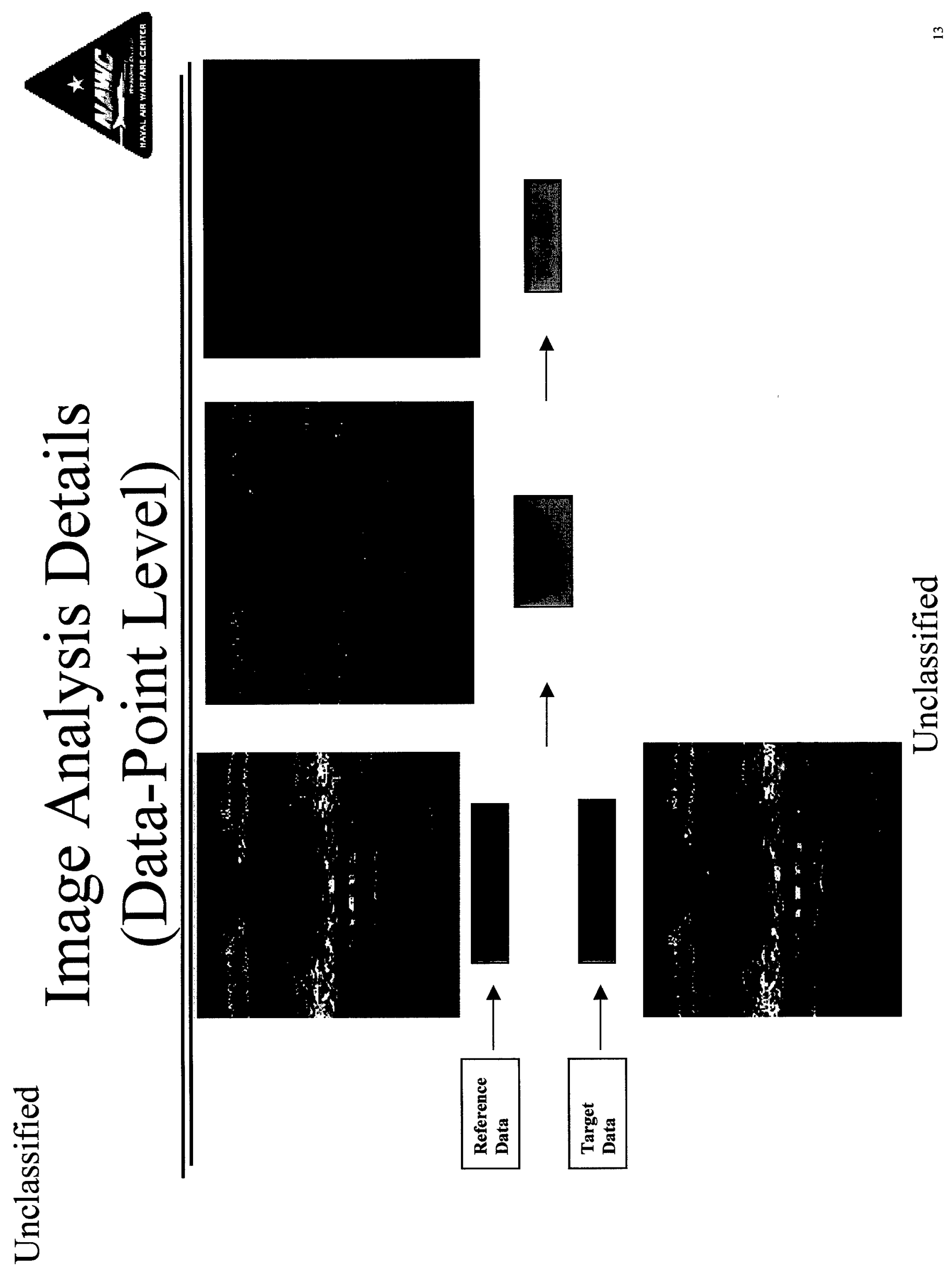



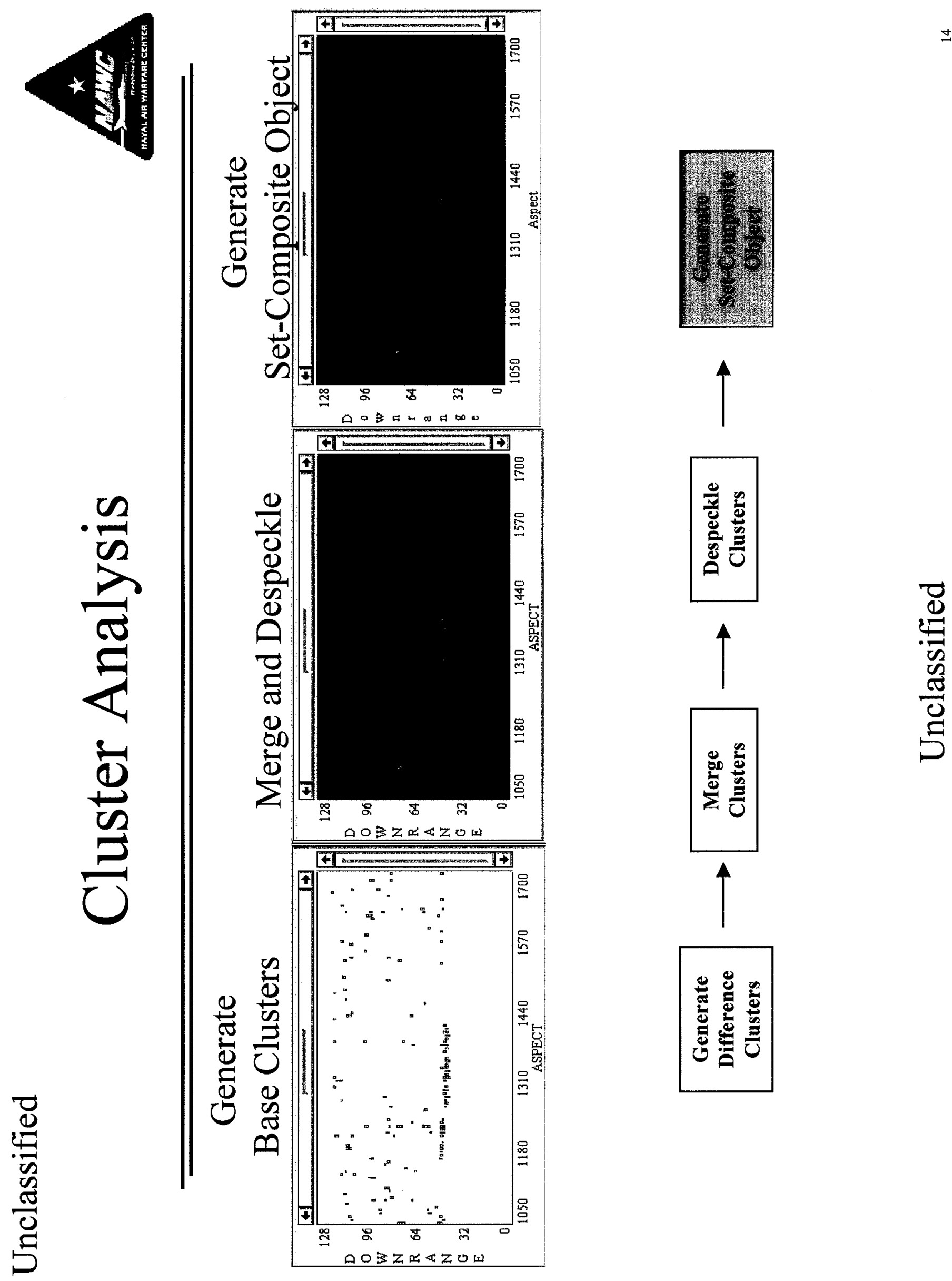

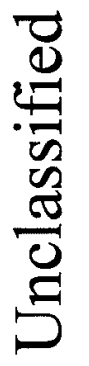




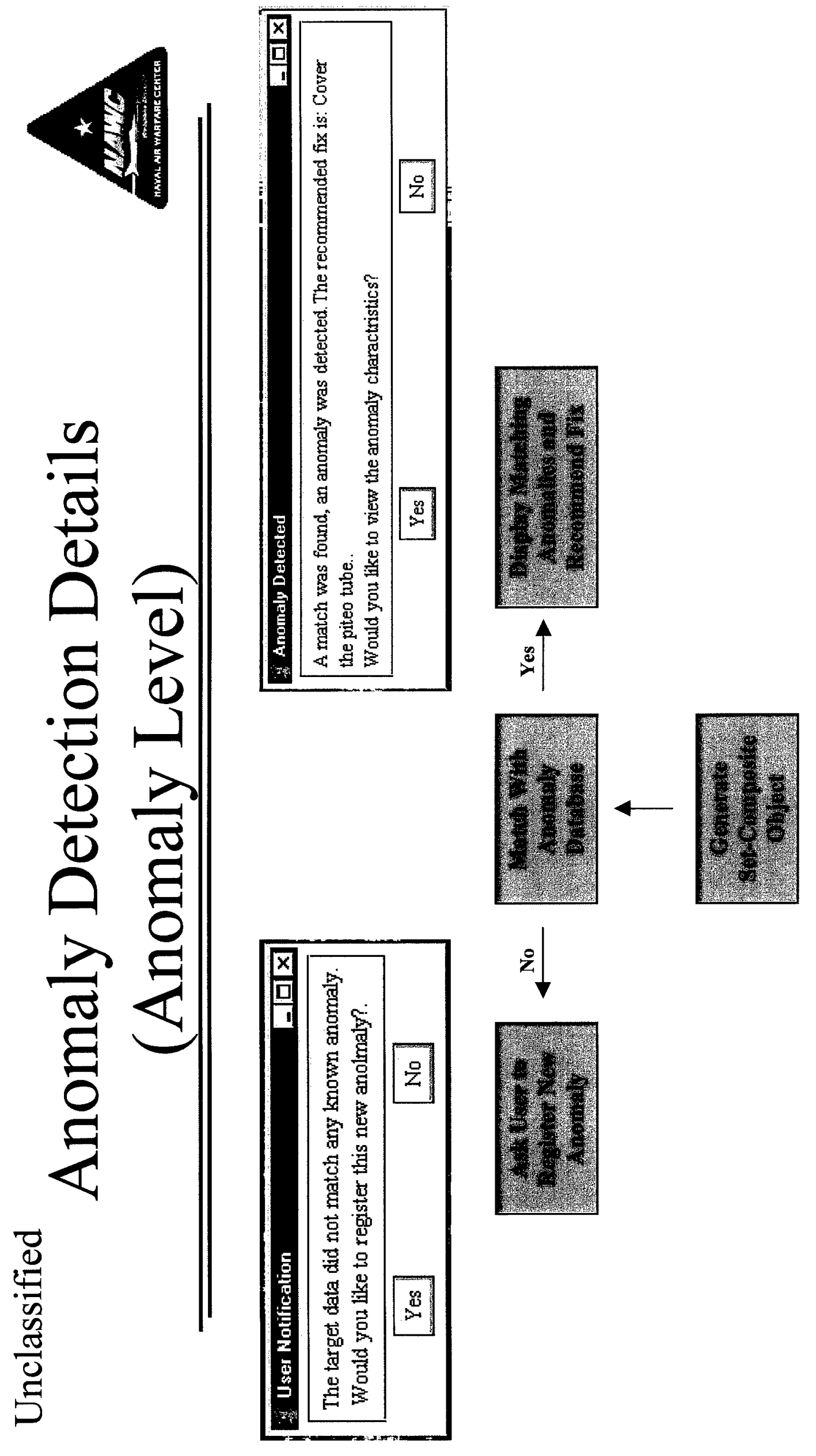

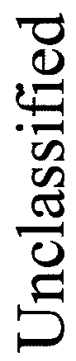




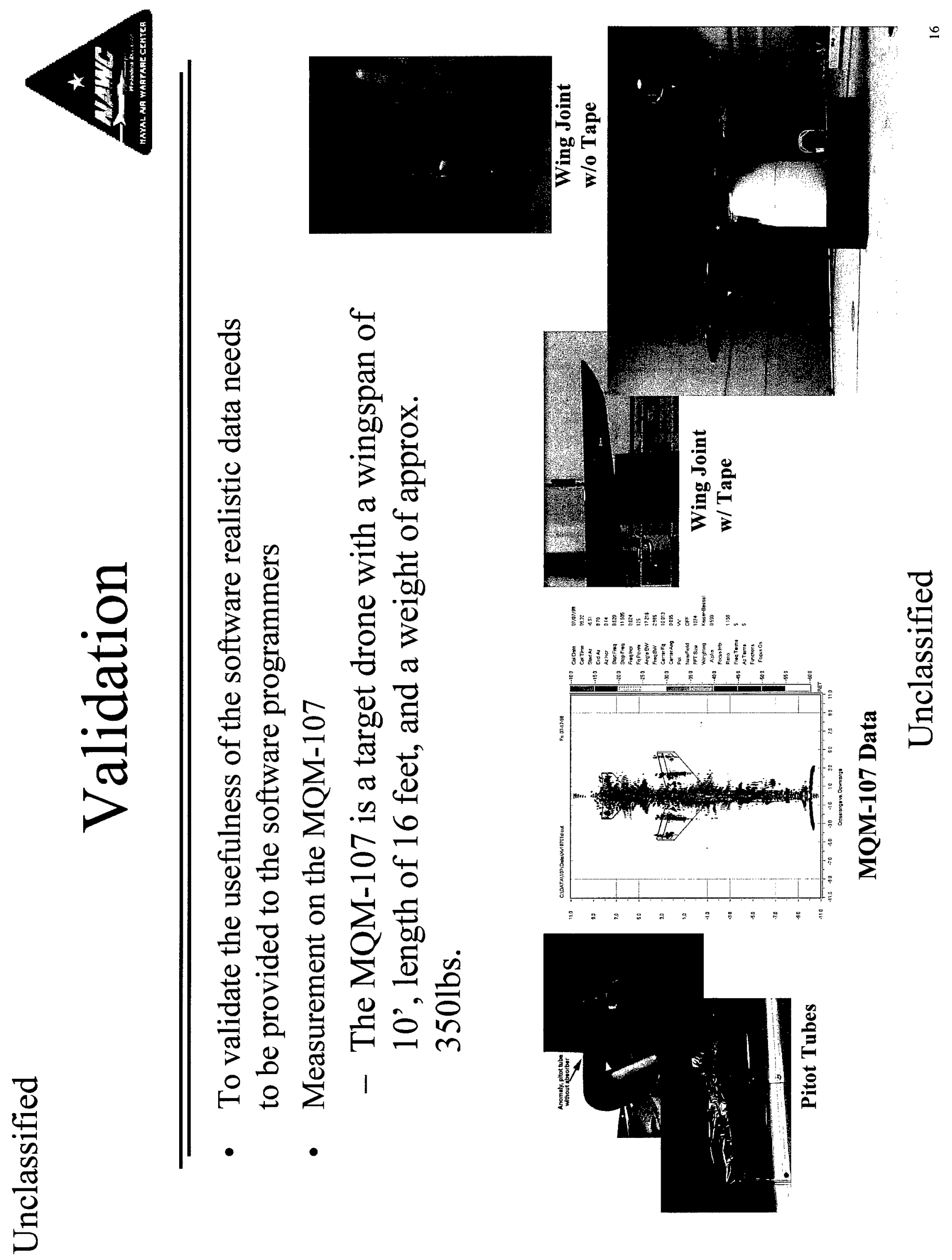



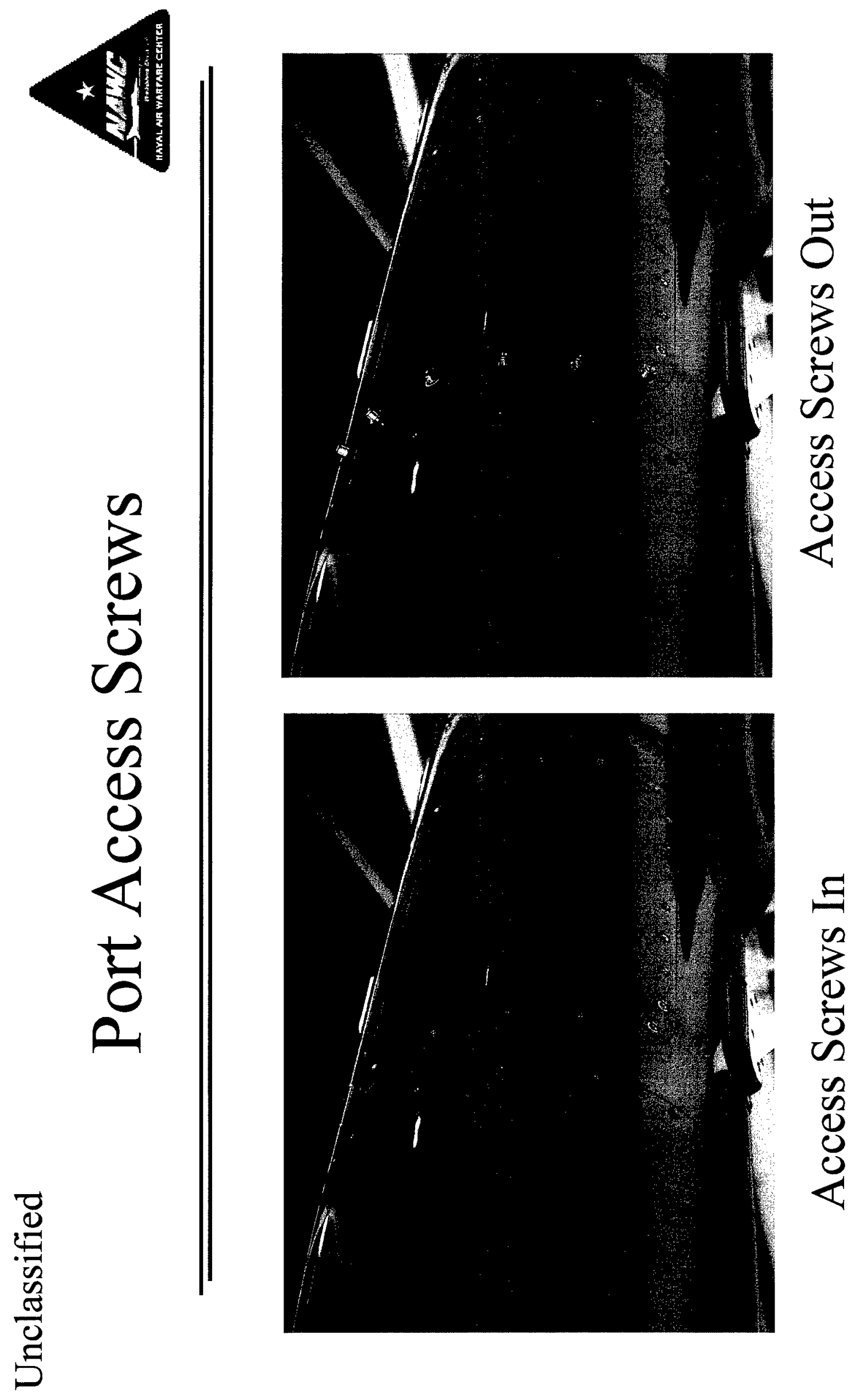

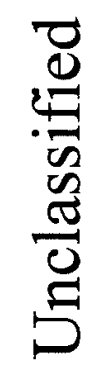

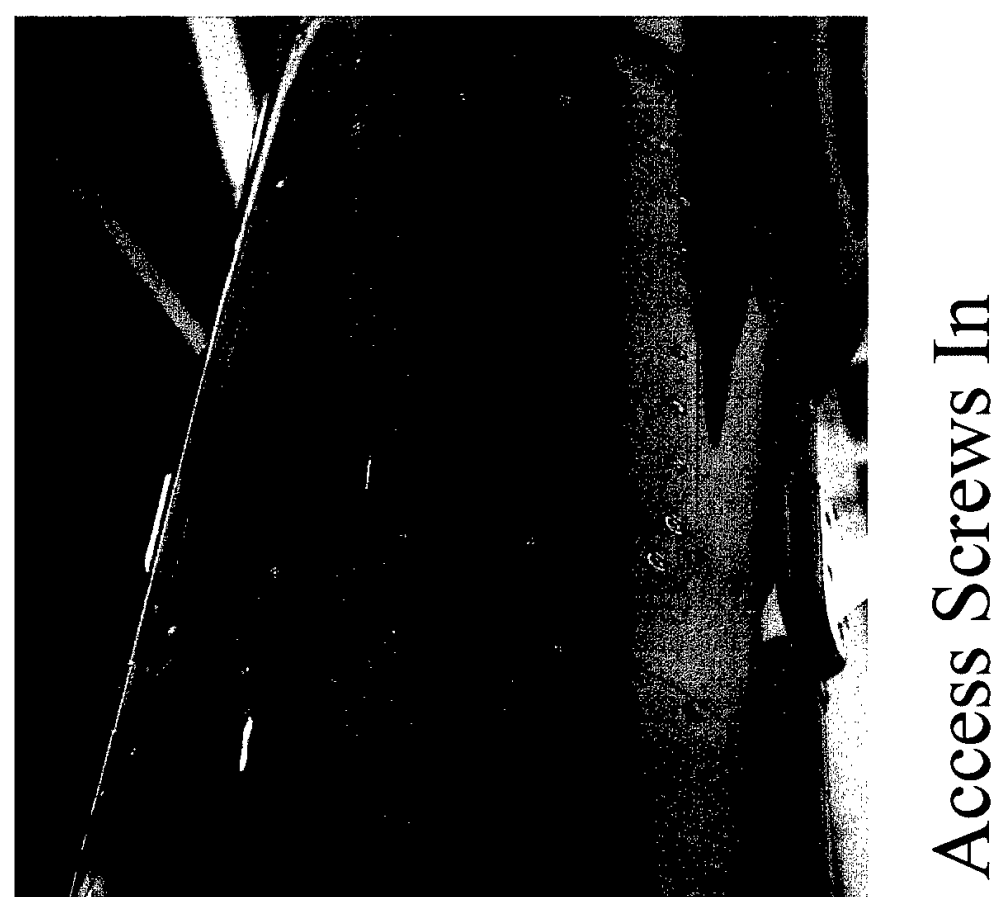




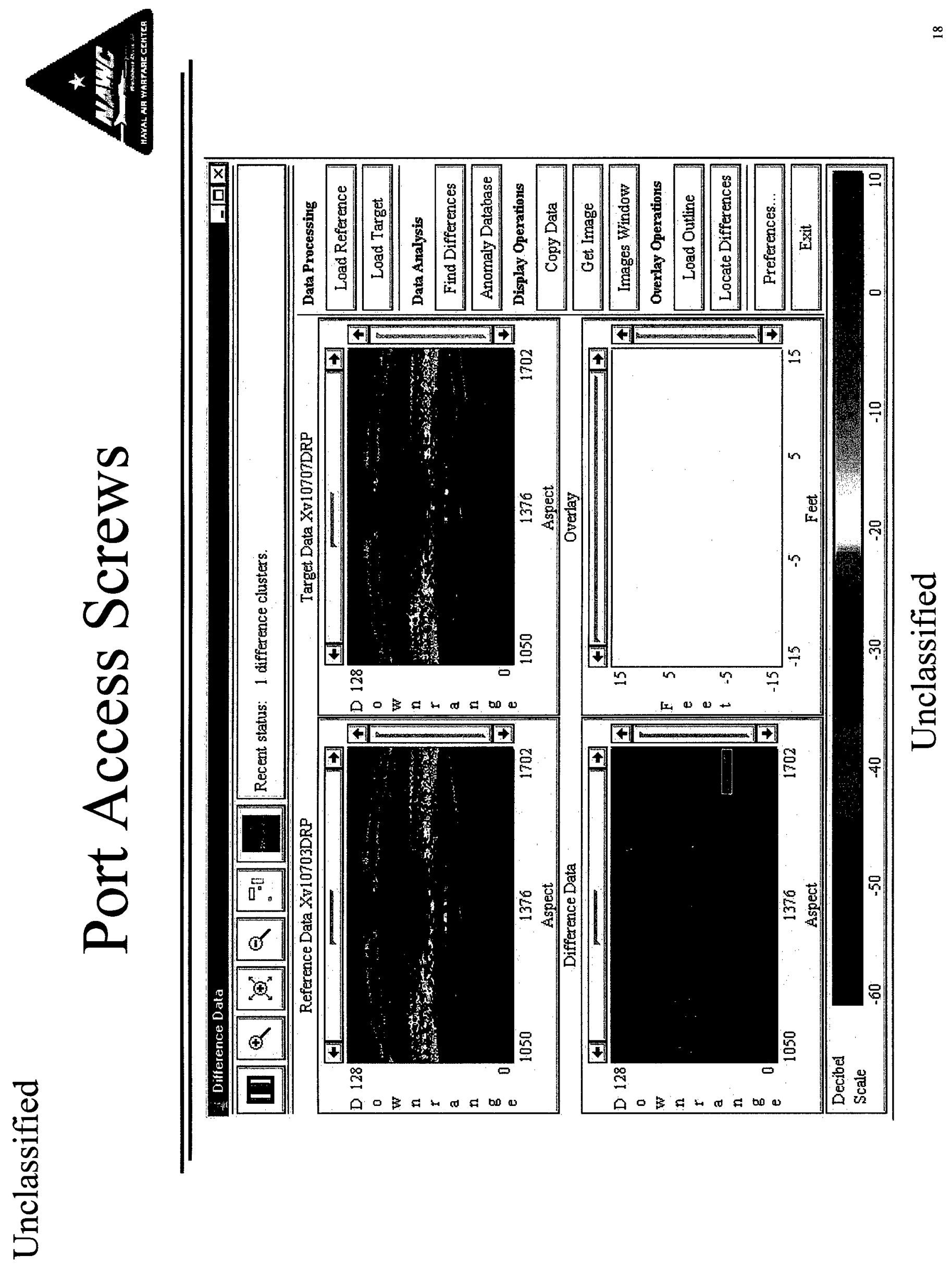




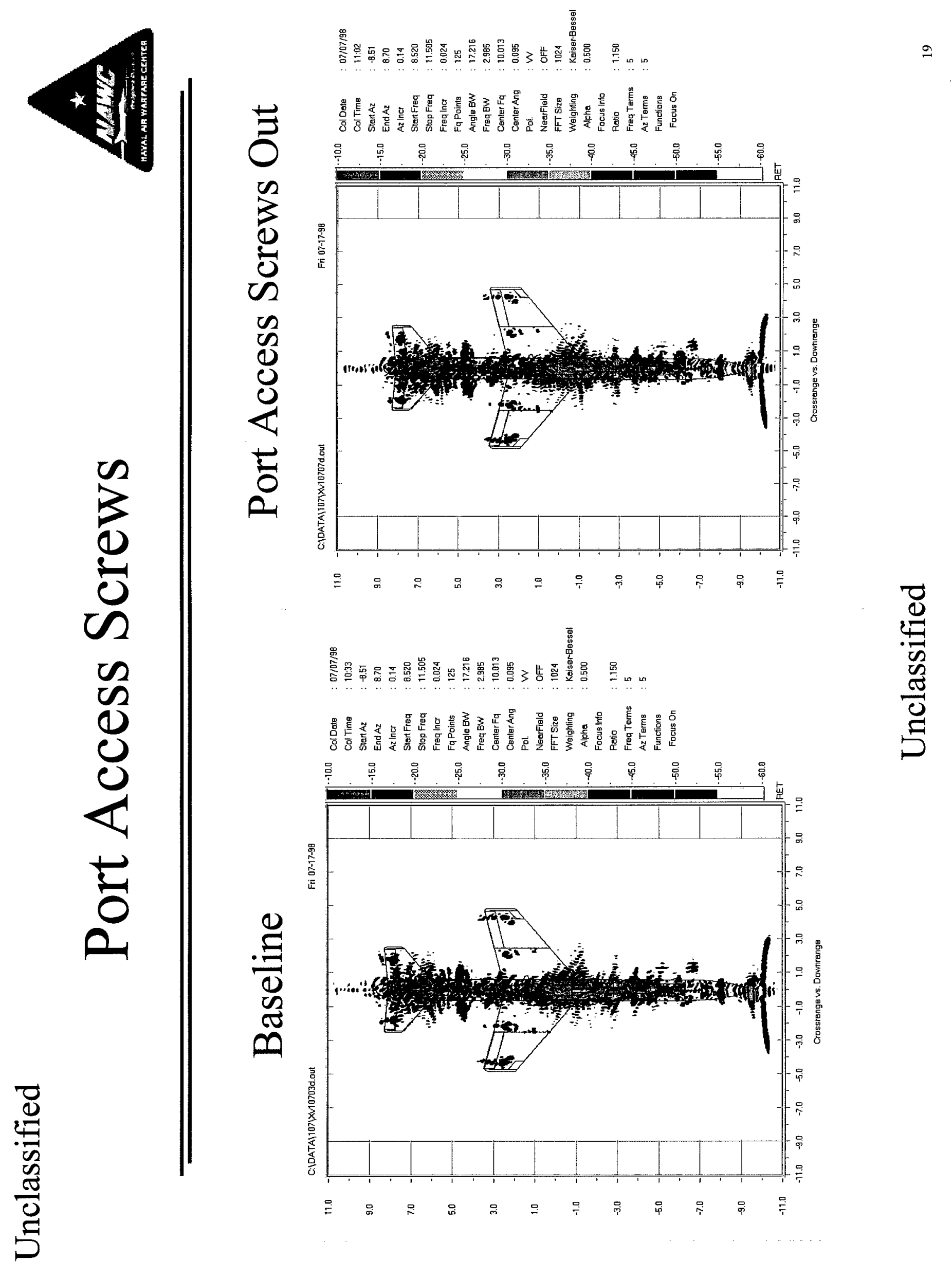



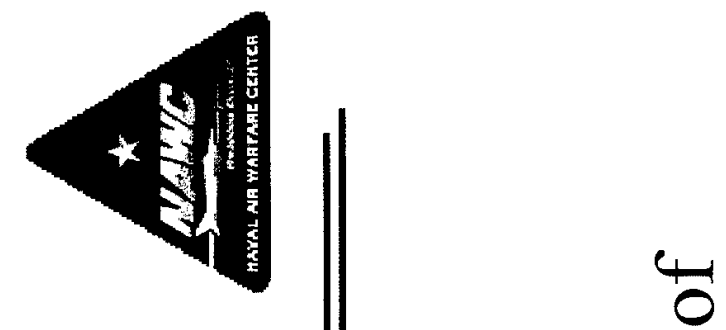

$\sum_{010}^{\infty}$

$\underset{0}{0} \stackrel{0}{0}$

$\frac{1}{6} \cdot \frac{1}{0}$

잉

82

$\rightarrow \frac{0}{2} \sum_{3}^{\infty}$

2

$\frac{0}{20}$

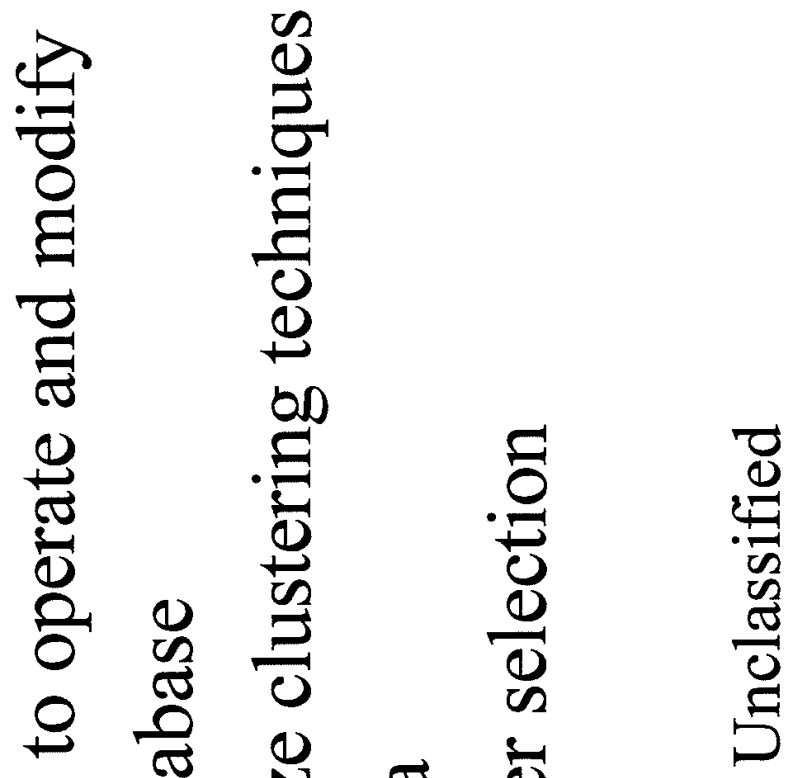

$P$ 光

$P \frac{0}{0}$ ब

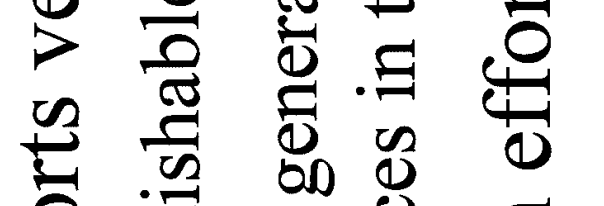

0 寻

ए क एव है

(1)

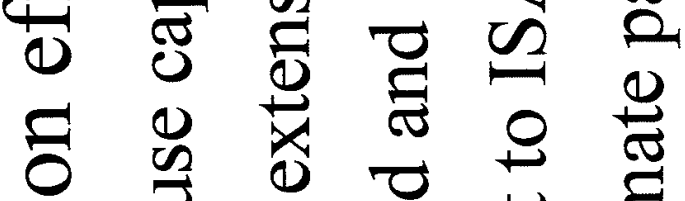

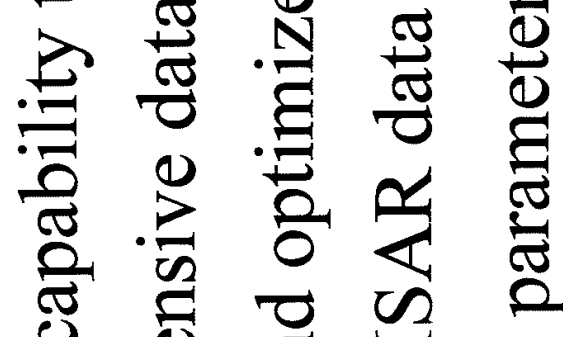

10

(1)

$+$

r

$\underset{\Xi}{\Xi}$

II

1 